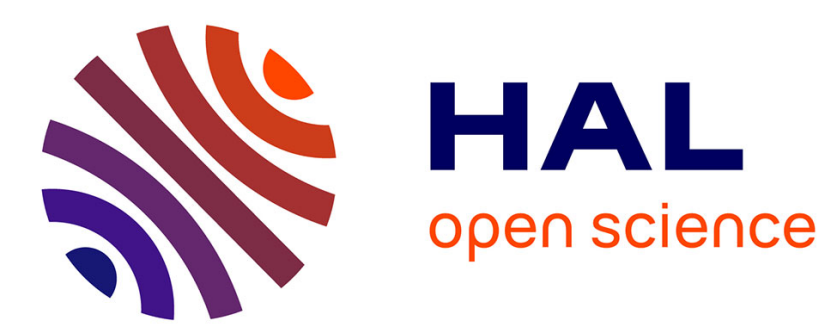

\title{
Improved slow light performances using photorefractive two-wave mixing
}

Nacera Bouldja, Marc Sciamanna, Delphine Wolfersberger

\section{To cite this version:}

Nacera Bouldja, Marc Sciamanna, Delphine Wolfersberger. Improved slow light performances using photorefractive two-wave mixing. Optics Letters, 2019, 44 (6), pp.1496. 10.1364/OL.44.001496 . hal-03223365

\section{HAL Id: hal-03223365 \\ https://hal-centralesupelec.archives-ouvertes.fr/hal-03223365}

Submitted on 11 May 2021

HAL is a multi-disciplinary open access archive for the deposit and dissemination of scientific research documents, whether they are published or not. The documents may come from teaching and research institutions in France or abroad, or from public or private research centers.
L'archive ouverte pluridisciplinaire HAL, est destinée au dépôt et à la diffusion de documents scientifiques de niveau recherche, publiés ou non, émanant des établissements d'enseignement et de recherche français ou étrangers, des laboratoires publics ou privés. 


\title{
Improved slow light performances using pho- torefractive two wave mixing
}

\author{
Nacera BOULDJA ${ }^{1,2, *}$, Marc SCIAMANNA ${ }^{1,2}$ and Delphine WOLFERSBERGER ${ }^{1,2}$ \\ ${ }^{1}$ Chair in Photonics, CentraleSupélec, LMOPS, F-57070 Metz, France \\ ${ }^{2}$ Université Lorraine,CentraleSupélec,LMOPS, F-57070 Metz, France \\ *nacera.bouldja@centralesupelec.fr
}

\begin{abstract}
We experimentally observe an ultra low effective group velocity of $0.9 \mathrm{~cm} / \mathrm{s}$ of light pulses using the two wave mixing process (TWM) in a SPS:Te crystal at visible wavelength. The time delay can be controlled through the nonlinear photorefractive gain and the input pulse duration. By optimizing the nonlinearity strength, we achieve a maximum fractional delay of $\mathbf{0 . 7 9}$ for pulse duration of $100 \mathrm{~ms}$. Our photorefractive slow-light system allows combining low group velocity with large delay-bandwidth product for pulse durations spanning three orders of magnitude.
\end{abstract}

ight slow-down has been first demonstrated in 1909 by Hendrik Lorentz [1], when he reported that the light group velocity in an atomic vapor can be smaller than the speed of light in the vacuum. Since then several studies have been performed in numerous dispersive materials to slow-down light pulses using various nonlinear effects. Applications include information processing, storage components [2] and interferometers [3].

The performance of a slow light system is typically measured through the value of the group velocity $v_{g}$, i.e the propagation speed of the light pulse envelope in the material. The control of the material dispersion through the gain spectrum is used to reduce the group velocity since:

$$
v_{g}=\frac{d}{\Delta \tau}=\frac{c}{n(\omega)+\omega \partial n / \partial \omega}
$$

where $\mathrm{d}$ is the thickness of the material, $\Delta \tau$ is the time delay, $n(\omega)$ is the refractive index, $\omega \partial n / \partial \omega$ is the material dispersion and $\omega$ the frequency detuning between the pulse and the pump. Another key parameter is the so-called fractional delay (FD), i.e the pulse delay $\Delta \tau$ divided by the full width at half maximum (FWHM) [4] at the slowlight system output. Since the pulse FWHM is inversely proportional to the signal bandwidth, the fractional delay is related to the so-called delay-bandwidth product (DBP) [5]. Ideally, applications seek significant reduction of $v_{g}$ with a large DBP value,or in other words, a significant delay for short input pulses and with little distortion of the pulses at the material output.

Slow light has been observed in numerous physical systems [6-9]. A milestone was achieved in 1999 [10] when Hau et al. reported a dramatic reduction of light speed down to $17 \mathrm{~m} / \mathrm{s}$ in an ultracold gas using the Electromagnetically Induced Transparency (EIT). Four years later, Podivilov et al. [11] demonstrated speed light reduction to $0.025 \mathrm{~cm} / \mathrm{s}$ through a two wave mixing process in $\mathrm{BaTiO}_{3}$ photorefractive crystal at room temperature. However, the material dispersion that leads to reduction of $v_{g}$ also significantly broadens the output pulse, hence limiting the FD value. In [11], FD is of the order of 0.4 for a pulse duration of $1.3 \mathrm{~s}$ in $\mathrm{BaTiO}_{3}$. Slowing down of $100 \mathrm{~ms}$ pulses was also obtained in a SPS crystal with $v_{g}=40 \mathrm{~cm} / \mathrm{s}$ and FD $=0.5$. Simultaneously, several other physical mechanisms including Brillouin [12,13] or Raman [14] scattering process in the optical fiber or propagation close to band-edge in photonic crystals [15] have shown the possibility to enhance the FD in a slow light system. For example, T. Baba et al. [15] measured to our knowledge the highest value of FD in the order of 57 in a photonic crystal coupled waveguide (PCCW). In these systems however the group velocity remains large $\left(v_{g}=11000 \mathrm{~km} / \mathrm{s}\right.$ in photonic crystal [15] and $v_{g}=12000 \mathrm{~km} / \mathrm{s}$ in optical fiber [16]) since an optimal pulse delay typically requires long material interaction length and this in addition introduces significant losses for the light pulses being slow-down.

It therefore appears current approaches remain unsuccessful in offering slow-down of short input light pulses while maintaining a small $v_{g}$, a large FD or, equivalently, little output pulse distortion.

In this letter, wCe optimize the experiment of two wave mixing in photorefractive material at room temperature. We specifically address the question of the slow light performance in terms of group velocity reduction, FD and input pulse duration. The choice of the SPS photorefractive crystal is justified by its large and broadband gain and its faster response time [17] compared to other ferroelectrics materials. SPS was also previously used for two-wave mixing experiments and slowing down of light $[18,19]$. While $v_{g}$ decreases monotonously with increasing the input pulse duration, the FD shows a maximum in a range of input pulse duration from $10 \mathrm{~ms}$ to $200 \mathrm{~ms}$ and remains large in a range of pulse durations spanning three orders of mag- 


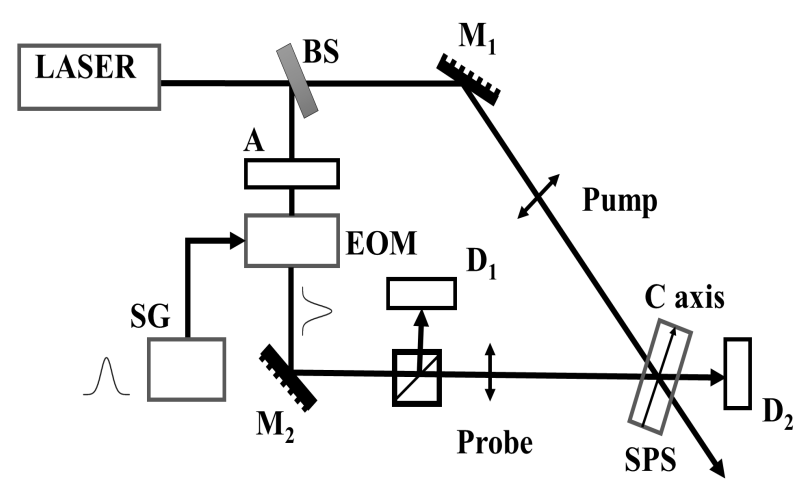

Figure 1: Slow light experimental setup ; EOM is the electrooptic modulator, $D_{i}$ are the detectors, $M_{i}$ are the mirrors, $B S$ are the beamsplitters.

nitude. Therefore, $100 \mathrm{~ms}$ pulses are slowed down with $v_{g} \sim 8 \mathrm{~cm} / \mathrm{s}$ and FD $\sim 0.8$, hence showing a significant improvement of slow-light performances when compared to [11]. Furthermore, our experience shows that the pulse is less distorted at the SPS crystal output than in $\mathrm{BaTiO}_{3}$.

Fig. 1 shows the experimental setup using a SPS crystal. The laser beam $(\lambda=638 \mathrm{~nm})$ with an intensity of 3.8 $W / \mathrm{cm}^{2}$ is split into two coherent beams, a signal $\mathrm{S}$ and a pump P with different powers, $17 \mathrm{~mW}$ and $40 \mathrm{~mW}$ for the signal and the pump respectively. The amplitude of the signal is modulated by an electro-optical modulator (EOM) placed before the mirror $M_{2}$. We use a signal generator and an amplifier as driver for the EOM to generate a Gaussian optical pulse shape. An attenuator (A) is placed before the modulator to vary the signal power at the crystal input face from $2 \mu \mathrm{W}$ to $3 \mathrm{~mW}$. The two-wave mixing gain is then measured for different input intensities $I_{0}=I_{s}(z=0)+I_{p}$, where $I_{p}$ and $I_{s}(z=0)$ are respectively the pump and signal input pulse intensities and $I_{p} \gg I_{s}$. The pump and the signal pulses enter on the SPS crystal through the zface with an intersection angle $2 \theta$. This SPS crystal with a thickness of $d=5 \mathrm{~mm}$, is grown in Institute of Solid State Physics and Chemistry, Uzhgorod National University by the conventional vapor-transport method [20]. It is known that the SPS crystal is characterized by a faster photorefractive build up time (of the order of ms) when compared to photorefractive $\mathrm{BaTiO}_{3}$ or $\mathrm{LiNbO}_{3}$ [21]. Finally, the input and output pulses are detected by two amplified silicon photodiodes $\left(D_{1}\right)$ and $\left(D_{2}\right)$.

The interference of two coherent waves in a photorefractive crystal induces the formation of a refractive index grating with a period $(\Lambda)$. When the light pulse and the pump pass through the SPS, they diffract on the grating and following Eq.(2) it results in the transfer of a part of the pump energy in the signal direction [11]:

$$
\left\{\begin{array}{r}
\frac{\partial A_{s}}{\partial z}=-i \frac{\pi n^{3} r}{\lambda} E A_{p} \\
\frac{\partial A_{p}}{\partial z}=\mp i \frac{\pi n^{3} r}{\lambda} E^{*} A_{s}
\end{array}\right.
$$

where $A_{s}$ and $A_{p}$ are respectively the amplitudes of the signal and the pump, $n$ is the refractive index of the crystal,
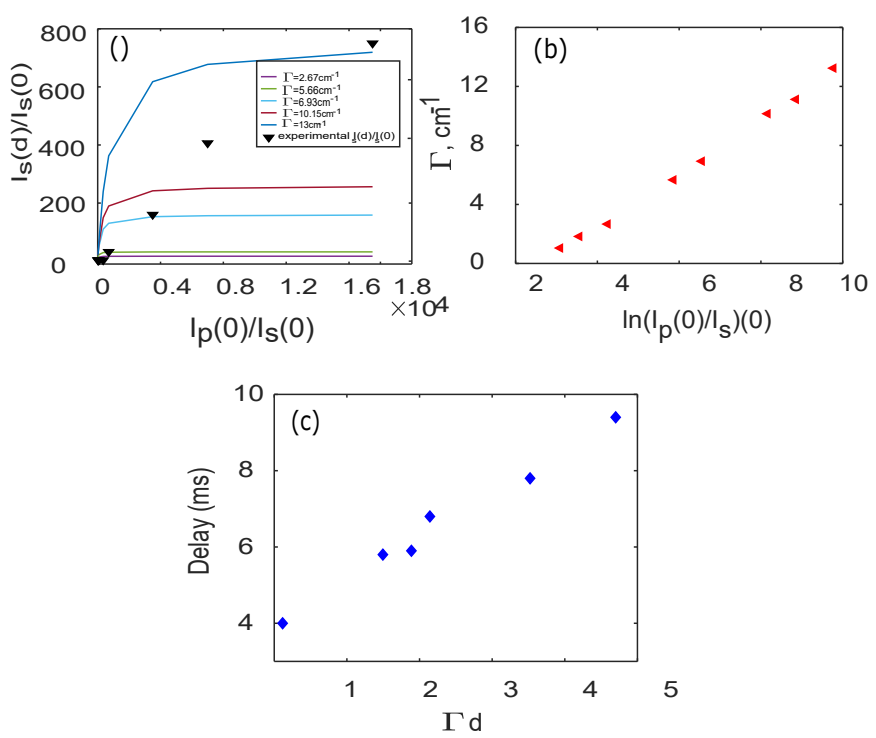

Figure 2: (a) Dependence $I_{s}(d) / I_{s}(0)$ on the input intensities ratio $\left(I_{p}(0) / I_{s}(0)\right)$ for different photorefractive gain values. $\mathbf{\nabla}$ are the operating points shown in (b). (b) Dependence of photorefractive gain $\Gamma$ input intensities ratio $\left(I_{p}(0) / I_{s}(0)\right)$ for grating period $\Lambda=$ $1.9 \mu \mathrm{m}$ and total input power $P_{0}=35 \mathrm{~mW}$. (c) Delay as a function of $\Gamma d$ for a crystal length $d=5 \mathrm{~mm}$ and input pulse duration of $10 \mathrm{~ms}$.

$r$ is the effective electro-optical coefficient, $E$ is the complex amplitude of the space charge field, $\lambda$ is the wavelength of the laser and $z$ is the propagation direction.

The measurement of the resulting amplification of the signal beam allows estimating the so-called photorefractive gain and relating this to the slow light performance. Indeed, if we call $\gamma=I_{s}(d) / I_{s}(0)$ the amplification factor, where $I_{s}(0)\left[I_{s}(d)\right]$ is the signal intensity at the entrance (output) of the crystal. and if we call $m=I_{p}(0) / I_{s}(0)$ the ratio of pump to signal intensity at crystal input, the photorefractive gain $\Gamma$ is related to $\gamma$ through the following Eq. (3), or equivalently Eq. (4) from Kukhtarev et al [22]:

$$
\begin{gathered}
\gamma=\frac{I_{s}(d)}{I_{s}(0)}=\frac{1+m}{1+m e^{-\Gamma d}} \\
\Gamma=\frac{1}{d} \ln \left(\frac{I_{s}(d)}{I_{p}(d)}\right)+\frac{1}{d} \ln \left(\frac{I_{p}(0)}{I_{s}(0)}\right)
\end{gathered}
$$

In our experiment, we fix $I_{p}(0)$ and vary $I_{s}(0)$ from $3 \mathrm{~mW}$ to $2 \mu W$. Fig. 2 (a) plots $\gamma$ versus $m$ for the corresponding $\Gamma$ in the optimal experimental conditions corresponding to $\Lambda=1.9 \mu \mathrm{m}$. Fig.2 (b) plots $\Gamma$ for increasing $m$. The photorefractive gain is therefore a quantity that varies with the operating conditions, in particular with $m$. In the following we operate at $m \geq 10^{4}$ which corresponds to what is called the undepleted pump approximation, for which the operating conditions are in the plateau of Fig.2 (a).

As shown in Fig.2(c) the measured photorefractive gain $\Gamma$ is related to the slow light performance and first of all to the pulse delay. We fix the input pulse duration to $10 \mathrm{~ms}$ and we measure the time delay $\Delta \tau$ (time delay between the maximum of the input and the output pulse) for different 
$\Gamma d$.As shown in Fig.2(c), the time delay increases with the photorefractive gain. for $\Gamma d=4.7$, the delay $\Delta \tau=9.4 \mathrm{~ms}$ is almost equal to the full input pulse duration $t_{0}$ at half level.

After characterizing the photorefractive gain and delay for an input pulse duration of $10 \mathrm{~ms}$, we perform a serie of measurements to determine the time delay, the output pulse duration and the delay-bandwidth product as a function of the input pulse duration in the optimal conditions. We fix the gain to $\Gamma=13 \mathrm{~cm}^{-1}$ corresponding to the maximum delay. Fig. 3 illustrates the temporal envelopes of the normalized output and input pulses as function of the time. For three input pulse durations $t_{0}$ of (a) $1.2 \mathrm{~ms}$ (shorter than the photorefractive response time $\tau=10 \mathrm{~ms}$ ), (b) 165 $m s$ and (c) $3.5 \mathrm{~s}$ (longer than the photorefractive response time), delays of $0.4 \mathrm{~ms}, 132 \mathrm{~ms}$ and $550 \mathrm{~ms}$ are measured respectively. The corresponding effective group velocities $v_{g}$ are computed from Eq.(5) and we obtain $12.5 \mathrm{~m} / \mathrm{s}, 2.1$ $\mathrm{cm} / \mathrm{s}$ and $0.9 \mathrm{~cm} / \mathrm{s}$ respectively.

$$
v_{g}=\frac{d(\mathrm{~cm})}{\Delta \tau(\mathrm{s})}
$$

By varying input pulse width between $0.3 \mathrm{~ms}$ and $3.5 \mathrm{~s}$, we can plot the experimental time delay and output pulses duration (FWHM) in Fig.4(a) and (b) respectively. As show in Fig.4(a), the time delay $\Delta \tau$ increases with the input pulse duration.The comparison of Fig.4(a) with Fig.5(b) from [23] tells us that: 1) we can slow-down pulses of durations smaller than $1 \mathrm{~ms}$ (here $300 \mu \mathrm{s}$ ) and of duration up to $3.5 \mathrm{~s}$. In [23] the shortest pulse for which slow light was recorded is of duration smaller than $150 \mathrm{~ms}$, and moreover fast and not slow light is observed for pulse duration above $200 \mathrm{~ms}$, 2) we achieve a larger delay than in [23] for pulse duration from $10 \mathrm{~ms}$ to $3.5 \mathrm{~s}$. For comparison, Ref [11] measured a group velocity of $40 \mathrm{~cm} / \mathrm{s}$ and Ref [23] achieved a delay $\Delta \tau=22 \mathrm{~ms}$ for a pulse duration of $150 \mathrm{~ms}$. For the same pulse duration we measure a delay of $78 \mathrm{~ms}$ which corresponds to a group velocity of the order of $6.4 \mathrm{~cm} / \mathrm{s}$; hence confirming an overall improvement of the slow light performances.

As mentioned earlier, it is interesting to analyze also the FD performance. According to previous studies, slowingdown of the group velocity and reaching a large FD simultaneously is difficult because of the output pulse distortion. Fig.4(c) plots the FD versus the input pulse duration. Although the experimental data are scattered significantly, the FD remains of the order of 0.5 in a broad range of input pulse duration. While scanning the input duration, a max FD of 0.79 is achieved for a pulse duration of 165 $m s$ (corresponding to Fig.3(b)). In Fig.4(c), we compare the achieved FD with the one retrieved from the data of ref [23]. Although the dependency of FD on the pulse duration remains qualitatively the same, the FD is systematically larger in the full range of pulse durations under analysis and spanning four orders of magnitude. Typically the FD is improved by a factor 2 when compared to [23]. In addition, we extend the analysis of [23] to light pulse durations below the millisecond time-scale.
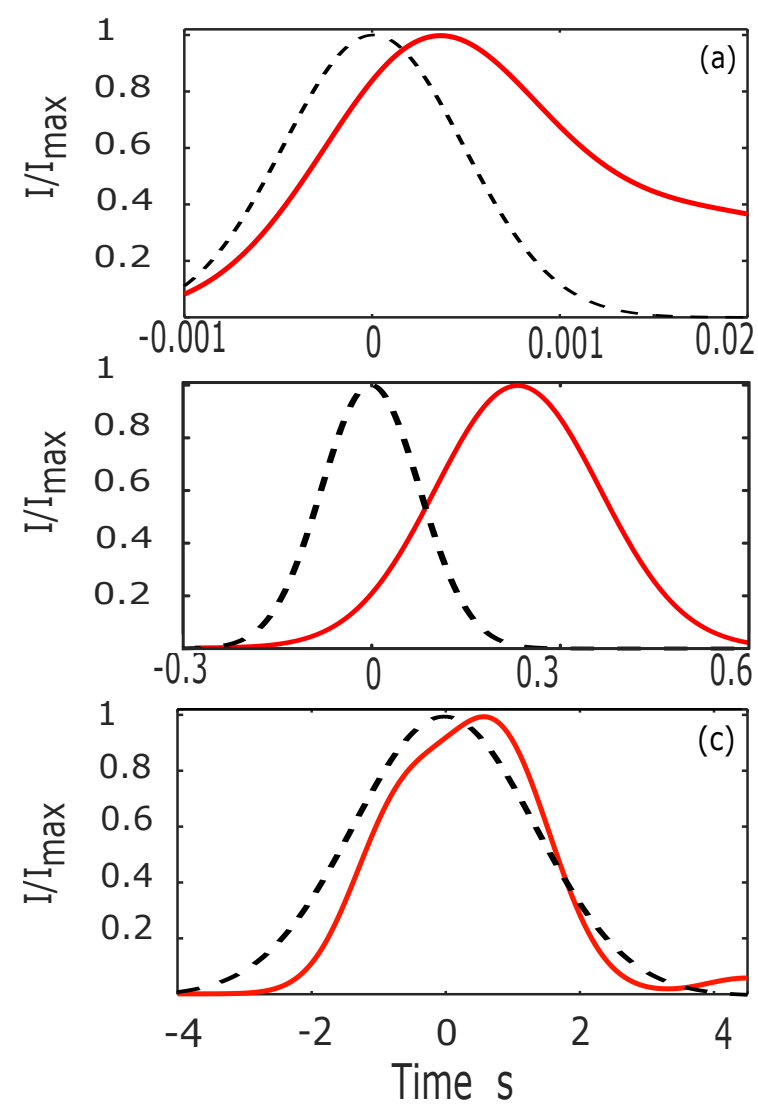

Figure 3: Temporal envelopes of the normalized input pulse (dotted line) and output pulse (red line) for three input pulse durations, (a) $t_{0}=1.2 \mathrm{~ms}$, (b) $t_{0}=165 \mathrm{~ms}$ and (c) $t_{0}=3.5 \mathrm{~s}$ corresponding to group velocities of $12.5 \mathrm{~m} / \mathrm{s}, 2.1 \mathrm{~cm} / \mathrm{s}$ and $0.9 \mathrm{~cm} / \mathrm{s}$ respectively, $\Gamma=13 \mathrm{~cm}^{-1}, \Lambda=1.9 \mu \mathrm{m}$ and $d=5 \mathrm{~mm}$.

Now, if we compare the output pulse shape to the input one, the result in Fig. 5 shows that the two-wave mixing in the SPS enables the slowdown of the output pulses without much distortion even for a strong gain. Fig. 5 tells us that the output pulse durations $t$ measured experimentally is close to ideal case $\left(t=t_{0}\right)$. Also, we note for some pulse durations, the compression of the pulse when it passes through the crystal.

We explain the small distortion by 1) the fact that gain is smaller than that used in [11], 2) the presence of two charge carriers in SPS that are responsible for a fast and a slow grating whose interplay allows to reduce the broadening of the output pulse [18]. By changing the rotation c-axis of the crystal, we modify the dispersion sign and the delayed light pulse can be accelerated. This result is shown in Fig.6.

To conclude, the photorefractive two-wave mixing experiment presented in this letter allows combining significant slow down of light pulses (below $\mathrm{cm} / \mathrm{s}$ ) and large fractional delay (close to 1 ) or equivalently large. We detail the dependency of both delay and FD on several experimental parameters such as the input intensity, the photorefractive gain and the duration of the pulse to be delayed. A large $\Gamma d$ induces typically a temporal deformation of the 


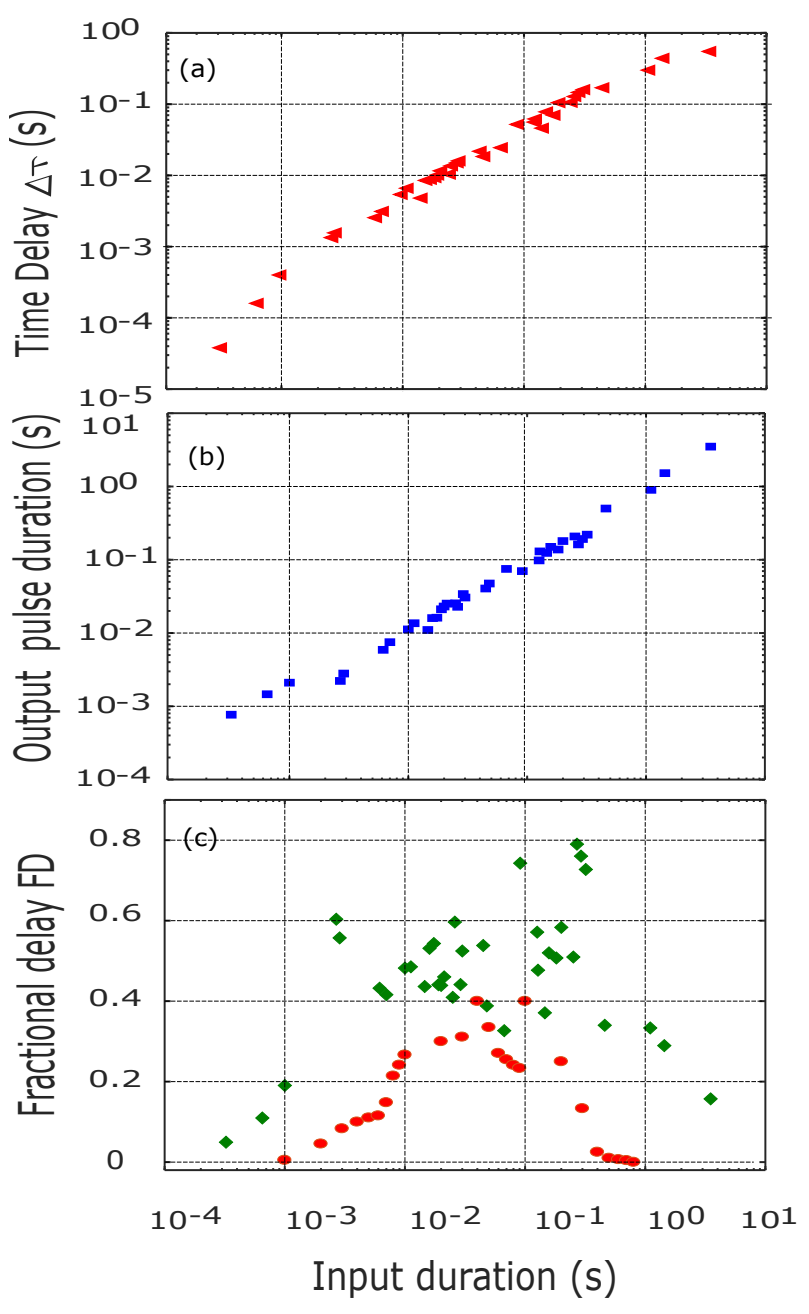

Figure 4: Pulse delay $\Delta \tau$ (a), full output width at half maximum $t$ (b) and delay bandwidth product (c) as function of the full input duration at half maximum $t_{0}, \Gamma d=6.6, \Lambda=1.9 \mu \mathrm{m}, d=5 \mathrm{~mm}$, $\tau=10 \mathrm{~ms}$. Red dot curve has FD calculated from the time delay values and output pulse durations of the publication [23].

output pulses which reduces FD. The distortion observed in our SPS crystal is however small compared to the one measured in other photorefractive crystals such as $\mathrm{BaTiO}_{3}$. The successful simultaneous achievement of a small group velocity below $\mathrm{cm} / \mathrm{s}$ and a FD close to 1 at room temperature is a significant improvement when compared to other slow-light systems.

We identify two paths for further improvement. First, the results are obtained at visible wavelengths, but the photorefractive gain in SPS is shown to be broadband up to at least $1.55 \mu \mathrm{m}$ [24]. The transparency window goes up to $8 \mu \mathrm{m}$ but there is not data yet of beam coupling experiences beyond $1.55 \mu \mathrm{m}$. We therefore expect similar performances can be achieved for slowing down light pulses at telecom wavelengths for large $\Gamma d$. Secondly, Ref [25] makes use of a pulses train to increase the overall interval intensity hence reducing the photorefractive response time and making it possible to slowdown very shorter pulses down to ps. It will be interesting to analyze FD performances for those

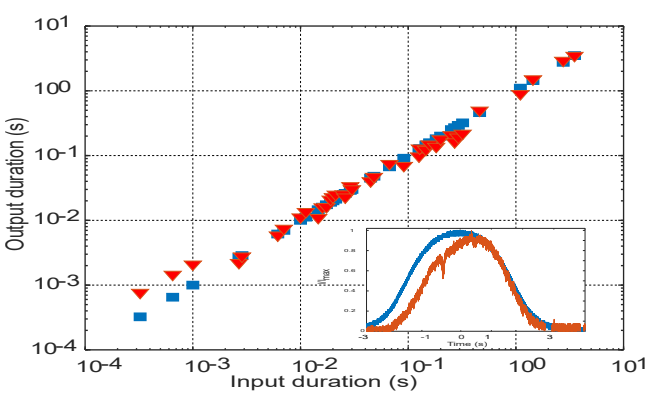

Figure 5: Output pulse duration $t$ (red triangle) as function of the input pulse duration $t_{0}, \Gamma d=6.6, \Lambda=1.9 \mu \mathrm{m}, \tau=10 \mathrm{~ms}$. A curve with blue squares plots the output pulse with no coupling in the crystal and therefore $t=t_{0}$.
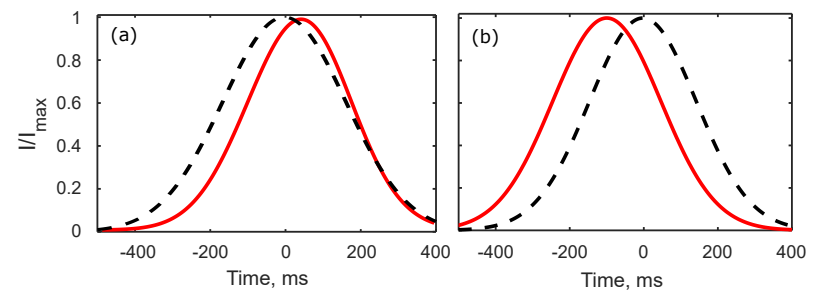

Figure 6: Temporal envelopes of the normalized input pulse (dotted line) and output pulse (red line) for input duration $t_{0}=380 \mathrm{~ms}$ (a) time delay $\Delta \tau=0.35 \mathrm{~ms}$, (b) time advance $\Delta \tau=-0.97 \mathrm{~ms}$ according to the dispersion sign, $\Gamma d=5$ and $\Gamma d=-5$ respectively.

short pulse durations.

\section{Acknowledgements}

The authors thank the founders of the Chair in Photonics: Ministry of Higher Education and Research, European Union (FEDER), Region Grand Est, Departement de la Moselle, Metz Metropole, Airbus-GDI Simulation, CentraleSupélec and Fondation Supélec. The authors also thank A.A. Grabar from the University of Uzhgorod for the SPS sample.

\section{References}

[1] H. A. Lorentz, The Theory of Electrons and Its Applications to the Phenomena of Light and Radiant Heat: A Course of Lectures Delivered in Columbia University, New York, in March and April, 1906. Columbia University Press, 1909, vol. 29.

[2] C. Liu, Z. Dutton, C. H. Behroozi, and L. V. Hau, "Observation of coherent optical information storage in an atomic medium using halted light pulses," Nature, vol. 409, no. 6819, p. 490, 2001.

[3] Z. Shi, R. W. Boyd, R. M. Camacho, P. K. Vudyasetu, and J. C. Howell, "Slow-light fourier transform interferometer," Phys. Rev. Lett., vol. 99, no. 24, p. 240801, 2007. 
[4] G. M. Gehring, H. Shin, R. W. Boyd, C.-M. Kim, and B. S. Ham, "Tunable optical time delay of quantum signals using a prism pair," Optics Express, vol. 18, no. 18, pp. $19156-19162,2010$.

[5] G. M. Gehring, R. W. Boyd, A. L. Gaeta, D. J. Gauthier, and A. E. Willner, "Fiber-based slow-light technologies," Journal of Lightwave Technology, vol. 26, no. 23, pp. 3752-3762, 2008.

[6] W. Horn, J. v Bassewitz, and C. Denz, "Slow and fast light in photorefractive sbn: 60," Journal of Optics, vol. 12, no. 10, p. 104011, 2010.

[7] L. Thévenaz, "Slow and fast light in optical fibres," Nature photonics, vol. 2, no. 8, p. 474, 2008.

[8] U. Bortolozzo, S. Residori, and J.-P. Huignard, "Slowlight through nonlinear wave-mixing in liquid crystal light-valves," Comptes Rendus Physique, vol. 10, no. 10, pp. 938-948, 2009.

[9] R. M. Camacho, M. V. Pack, J. C. Howell, A. Schweinsberg, and R. W. Boyd, "Wide-bandwidth, tunable, multiple-pulse-width optical delays using slow light in cesium vapor," Phys. Rev. Lett., vol. 98, no. 15, p. 153601, 2007.

[10] L. V. Hau, S. E. Harris, Z. Dutton, and C. H. Behroozi, "Light speed reduction to 17 metres per second in an ultracold atomic gas," Nature, vol. 397, no. 6720, p. 594, 1999.

[11] E. Podivilov, B. Sturman, A. Shumelyuk, and S. Odoulov, "Light pulse slowing down up to $0.025 \mathrm{~cm} / \mathrm{s}$ by photorefractive two-wave coupling," Phys. Rev. Lett., vol. 91, p. 083902, Aug 2003. [Online]. Available: https://link.aps.org/doi/10. 1103/PhysRevLett.91.083902

[12] L. Thevenaz, S.-H. Chin, K.-Y. Song, and M. GonzalezHerraez, "Flexible slow and fast light in optical fibers," in Lasers and Electro-Optics Society, 2006. LEOS 2006. 19th Annual Meeting of the IEEE. IEEE, 2006, pp. 18-19.

[13] L. Yi, L. Zhan, W. Hu, and Y. Xia, "Delay of broadband signals using slow light in stimulated brillouin scattering with phase-modulated pump," IEEE Photonics Technology Letters, vol. 19, no. 8, pp. 619-621, 2007.

[14] J. Liang, M. Katsuragawa, F. Le Kien, and K. Hakuta, "Slow light produced by stimulated raman scattering in solid hydrogen," Phys. Rev. A., vol. 65, no. 3, p. 031801, 2002.

[15] T. Baba, T. Kawasaki, H. Sasaki, J. Adachi, and D. Mori, "Large delay-bandwidth product and tuning of slow light pulse in photonic crystal coupled waveguide," Optics express, vol. 16, no. 12, pp. 9245-9253, 2008.
[16] Y. Okawachi, M. S. Bigelow, J. E. Sharping, Z. Zhu, A. Schweinsberg, D. J. Gauthier, R. W. Boyd, and A. L. Gaeta, "Tunable all-optical delays via brillouin slow light in an optical fiber," Phys. Rev. Lett., vol. 94, no. 15, p. 153902, 2005.

[17] T. Bach, M. Jazbinšek, G. Montemezzani, P. Günter, A. A. Grabar, and Y. M. Vysochanskii, "Tailoring of infrared photorefractive properties of sn 2 p 2 s 6 crystals by te and sb doping," JOSA B, vol. 24, no. 7, pp. 1535-1541, 2007.

[18] B. Sturman, P. Mathey, and H.-R. Jauslin, "Slowdown and speedup of light pulses using the selfcompensating photorefractive response," JOSA $B$, vol. 28, no. 2, pp. 347-351, 2011.

[19] A. Shumelyuk, K. Shcherbin, S. Odoulov, B. Sturman, E. Podivilov, and K. Buse, "Slowing down of light in photorefractive crystals with beam intensity coupling reduced to zero," Physical review letters, vol. 93, no. 24, p. 243604, 2004.

[20] R. Nitsche and P. Wild, "Crystal growth of metalphosphorus-sulfur compounds by vapor transport," Materials Research Bulletin, vol. 5, no. 6, pp. 419-423, 1970.

[21] A. A. Grabar, M. Jazbinšek, A. N. Shumelyuk, Y. M. Vysochanskii, G. Montemezzani, and P. Günter, "Photorefractive effects in sn 2 p 2 s 6," in Photorefractive Materials and Their Applications 2. Springer, 2007, pp. 327-362.

[22] N. Khuktarev, V. Markov, S. Odulov, M. Soskin, and V. Vinetskii, "Holographic storage in electrooptic crystals. ii. beam coupling - light amplification," Ferroelectrics, vol. 22, pp. 961-964, 1979.

[23] A. Shumelyuk and S. Odoulov, "Light pulse manipulation in sn2p2s6," Journal of Optics, vol. 12, no. 10, p. $104015,2010$.

[24] R. Mosimann, P. Marty, T. Bach, F. Juvalta, M. Jazbinsek, P. Günter, and A. A. Grabar, "High-speed photorefraction at telecommunication wavelength 1.55 $\mu \mathrm{m}$ in sn 2 p 2 s 6: Te," Optics letters, vol. 32, no. 22, pp. 3230-3232, 2007.

[25] T. Bach, K. Nawata, M. Jazbinšek, T. Omatsu, and P. Günter, "Optical phase conjugation of picosecond pulses at $1.06 \mu$ in sn 2 p 2 s 6 : Te for wavefront correction in high-power nd-doped amplifier systems," Optics express, vol. 18, no. 1, pp. 87-95, 2010. 